\title{
Strates
}

STRATES Matériaux pour la recherche en sciences sociales

14 | 2008

Espaces du quotidien

\section{Reconstruction territoriale et pratiques quotidiennes liées à l'eau à Phnom Penh (Cambodge)}

\section{Céline Pierdet}

\section{(2) OpenEdition}

Journals

Édition électronique

URL : http://journals.openedition.org/strates/6675

DOI : $10.4000 /$ strates.6675

ISSN : $1777-5442$

Éditeur

Laboratoire Ladyss

Édition imprimée

Date de publication : 1 janvier 2008

Pagination : 101-116

ISBN : 0768-8067

ISSN : 0768-8067

Référence électronique

Céline Pierdet, « Reconstruction territoriale et pratiques quotidiennes liées à l'eau à Phnom Penh (Cambodge) », Strates [En ligne], 14 | 2008, mis en ligne le 04 mars 2013, consulté le 08 septembre 2020. URL : http://journals.openedition.org/strates/6675; DOI : https://doi.org/10.4000/strates.6675

Ce document a été généré automatiquement le 8 septembre 2020

Tous droits réservés 


\title{
Reconstruction territoriale et pratiques quotidiennes liées à l'eau à Phnom Penh (Cambodge)
}

\author{
Céline Pierdet
}

\section{NOTE DE L'ÉDITEUR}

Les photos sont de l'auteur.

1 Contrairement à d'autres capitales fluviales du Sud-Est asiatique telles que Hanoï ou Bangkok, Phnom Penh dispose de larges quais ouverts sur une vaste confluence de voies fluviales que sont les bras du Mékong, le Tonlé Sap ${ }^{1}$ et le Tonlé Bassac. Au Cambodge, quatre habitants sur cinq vivent encore à la campagne ${ }^{2}$ et la population de la capitale elle-même est d'origine majoritairement rurale. Un génocide, faisant près de 1,7 million de victimes entre 1975 et 1979, a surtout frappé les citadins et les élites urbaines. Mais l'arrivée d'experts occidentaux, le renouveau du tourisme et l'émergence d'une société de consommation ont favorisé la coexistence de pratiques agraires et citadines dans la capitale fluviale d'un pays où les berges sont traditionnellement dévolues aux activités maraîchères et à la pêche. Un même espace supporte désormais plusieurs formes de territorialisations. 
Pratiques agraires et citadines à Phnom Penh.

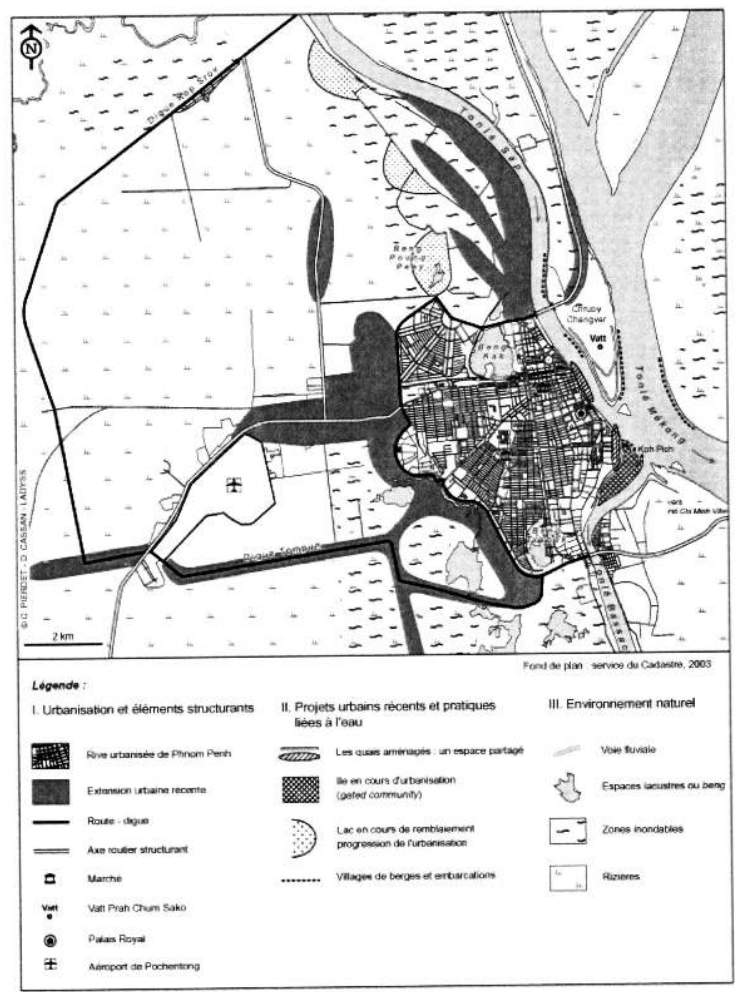

2 Depuis 1993, plusieurs projets urbains ont modifié la physionomie des berges de la capitale, sous-tendus par des préoccupations à la fois symboliques et de modernité. Des formes d'occupation spécifiques liées à l'eau, anciennes ou plus récentes, caractérisées par un rythme en apparence quotidien et quasi immuable, s'y côtoient. Mais des territorialités agraires sont aussi différenciées par l'appartenance ethnique des populations et cette quotidienneté est fortement pondérée par l'alternance saison sèche/saison des pluies, caractéristique de l'Asie des Moussons. Enfin, dans ce processus de reconstruction territoriale mis en œuvre depuis le début des années 1990, des formes nouvelles de territorialités sont désormais décelables aux marges des quartiers centraux de la ville, produits des nouveaux enjeux spatiaux et des conflits d'usages que cristallisent ces territoires encore ruraux ou en cours de transition vers la citadinité. Il s'agit donc d'analyser les formes de partage, de coexistence et de conflits entre territorialités traditionnelles et territorialités émergentes liées à l'eau à Phnom Penh $^{3}$.

Comment ces projets urbains, se voulant porteurs de modernité, ont-ils engendré une recomposition des territorialités traditionnelles, mettant ainsi en contact des pratiques d'origine agraire qui perdurent, mais sont de plus en plus à l'état de survivance, et des pratiques citadines nouvelles, symboliques de l'émergence récente d'une société de consommation, mais aussi d'une marginalisation accrue du plus grand nombre? Quelle construction territoriale en résulte-t-il ? 


\section{Les quais de Phnom Penh entre territorialités et temporalités multiples}

4 L'observation du quai Sisowath, situé au centre de la ville et de ce fait très fréquenté par les touristes, en différents moments de la journée, permet d'appréhender la diversité des formes d'occupation de l'espace liées à l'eau qui coexistent et se succèdent au quotidien sur cet espace linéaire. Ces territorialités, en cours de reconstruction depuis le début des années 1990, sont néanmoins pondérées par le rythme des saisons.

\section{De jour comme de nuit : un espace commun partagé}

Le long du Tonlé Sap, on peut identifier différentes temporalités, chacune marquée par des activités et des pratiques particulières, selon un rythme quotidien, que l'on a parfois réussi à ancrer dans des moments de vie. Ces quais, qui jouxtent les pelouses de la vaste esplanade du palais royal et qui sont ombragés par des kapokiers, articulent à la fois des activités proprement liées à la ville et des formes de pratiques en rapport avec l'eau, le fleuve. Ils concentrent donc une très forte diversité de formes d'occupation, ce qui leur donne l'aspect d'un espace souvent confus, aux pratiques imbriquées, mais toujours coloré, animé. À la fois lieux d'échange, de commerce, lieux de vie à part entière des plus pauvres attirés par la présence des touristes, mais aussi lieux de pratique d'activités sportives, de loisirs, les quais sont des territoires communs partagés, de jour comme de nuit.

Le quai Sisowath en début de matinée

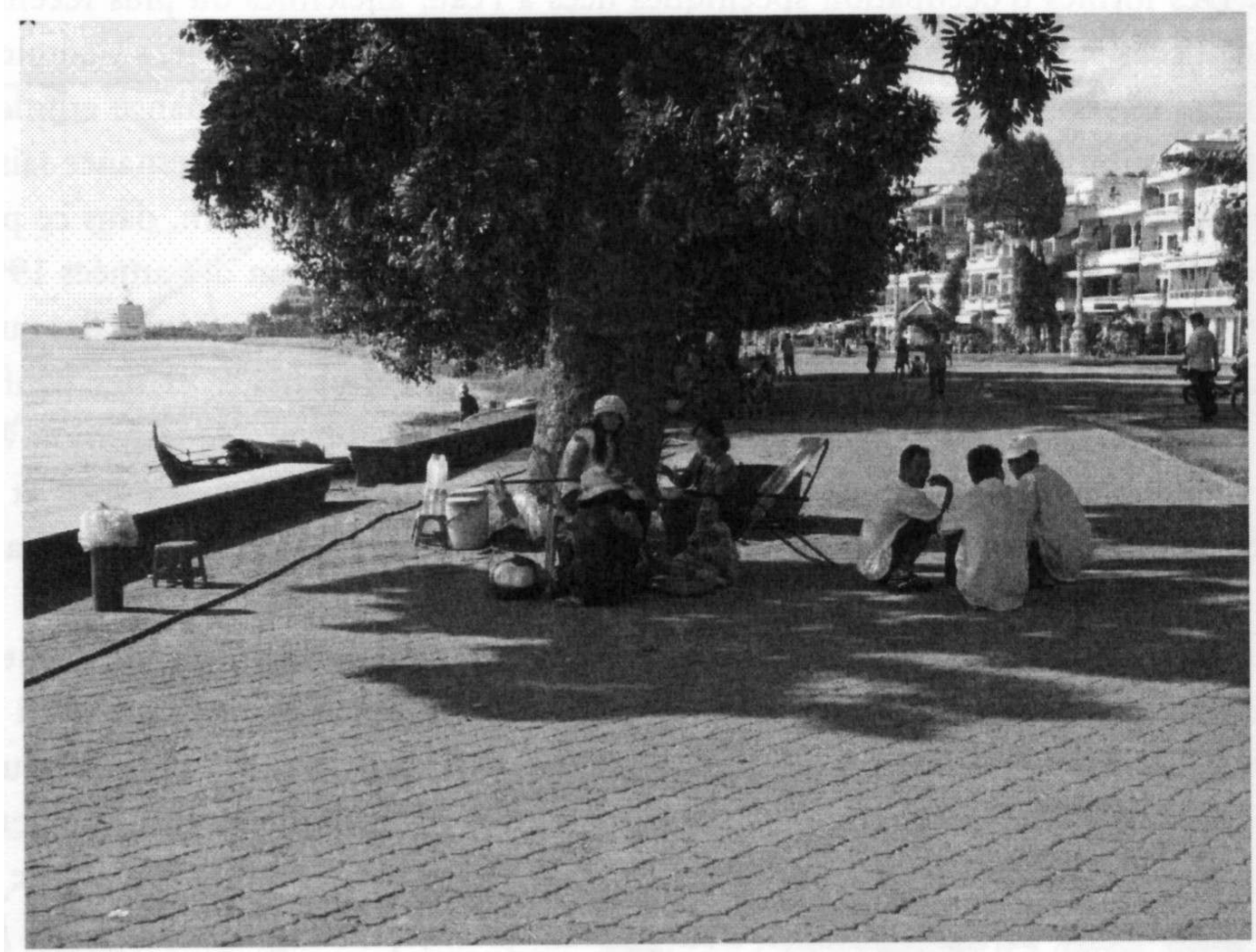

(c) CP, septembre 2003. 

royal, avant l'arrivée des premiers Phnompenhois qui viennent faire leurs exercices matinaux. Alors que le soleil se lève, des groupes se constituent. Une séance d'aérobic débute, tandis que d'autres effectuent un peu plus loin des mouvements de Tai Chi, avec lenteur et précision. Des individus isolés font leur jogging au milieu de cette foule déjà dense. Un professeur indonésien a créé une chorégraphie mêlant danses traditionnelles indonésiennes et aérobic. Arrivé à Phnom Penh en 1993 pour travailler à l'ambassade indonésienne, il a quitté son poste en 2000 pour ouvrir le «Bali Café » au bord du fleuve. Il a alors organisé cette activité matinale qui connaît un franc succès auprès des jeunes et des moins jeunes. D'autres, installés un peu à l'écart sur un tabouret en plastique, discutent entre eux. L'analyse de ces pratiques illustre bien le propos suivant : «Conscience de l'espace, la territorialité est avant tout conscience de soi et des autres en fonction des pratiques quotidiennes qui ne se réduisent pas à l'aire de résidence. [...] Pour le citadin, le quartier n'en constitue plus le référentiel fondamental ; c'est la ville, miroir de la société, avec son centre fonctionnel mais aussi ludique » (Bailly, 1982, p. 373). Ainsi, la traditionnelle pratique matinale d'arts martiaux en Asie, demeurée pendant longtemps dans la sphère privée, s'exerce-t-elle désormais quotidiennement en plein air, et engendre-t-elle une mobilité des plus aisées à l'échelle de la ville. À Ho-Chi-Minh-Ville, les Vietnamiens pratiquent le Viêt Vo Dao dans les grands parcs internes au tissu urbain dont dispose la métropole du Sud-Vietnam. À Phnom Penh, le Tai-chi-chuan, auquel d'autres sports modernes tels que le fitness sont venus se greffer au sein de cet espace, est ici pratiqué sur l'esplanade du palais royal, face à la confluence des Quatre-Bras.

6h30 et 7h00, les bonzes sortent du Vatt Unnalom en formant une file orangée, tandis que la foule se disperse pour aller travailler. Les quais se vident. Des populations «marginales» prennent alors possession des espaces publics: enfants des rues, mendiants mutilés, mais aussi petites vendeuses du secteur informel. Leur présence dans des lieux fortement fréquentés par les touristes, à partir de 8 h00 du matin, repose donc sur une négociation avec les forces de l'ordre. En échange d'un bakchich donné chaque mois aux policiers en faction devant le palais royal ou sur les quais, elles ont alors le droit de s'approprier un emplacement. Assis sur un carton ou sur un tabouret, éleveurs d'oiseaux, vendeuses de fleurs de lotus ou de brioches, peuvent étaler sur une natte ou disposer dans des corbeilles les produits qu'ils ont à vendre.

Chacun n'occupe guère plus de $1,5 \mathrm{~m}^{2}$ au sol. Après leur départ, il ne restera aucune trace de leur passage. Pratiques temporaires, parfois fugitives, mais sans cesse renouvelées, heure après heure, jour après jour, faites d'interrelations qui participent de la construction de cet espace en territoire.

Les quais restent le lieu privilégié des bars, des restaurants et des hôtels. L'agitation matinale est déjà loin quand les premiers touristes en sortent. Mais les bords du fleuve restent aussi un lieu de sortie très prisé des Cambodgiens. Sur les nouveaux quais situés dans l'axe du monument de l'Indépendance plus au sud, dès la sortie du travail, ils viennent s'asseoir sur le parapet, pique-niquer en famille en s'installant sur des nattes, sur les pelouses, ou se promener au bord de l'eau. Une fête foraine y est installée en permanence. Les vendeurs ambulants, telle cette marchande de pop-corn, en profitent, même si les policiers les rackettent. De nouvelles baraques en bois, dotées de hamacs et de téléviseurs, diffusant sketches comiques et karaoké, essaient d'attirer des clients.

Strates, $14 \mid 2008$ 
À partir de 20h00, l'espace se scinde plus nettement : d'un côté, les touristes sortis pour dîner, qui remplissent les terrasses sur le trottoir occidental, illuminé par les restaurants ; sur le bord du fleuve au contraire, le monde de la survie s'organise, dans une certaine pénombre. Un homme distribue à des enfants des bouquets de fleurs qu'ils devront vendre dans la soirée. Pour ces enfants des rues, les quais qu'ils se partagent sont quasiment le seul espace pratiqué, celui dont ils tirent leurs maigres revenus. Les bambins commencent alors à circuler d'un restaurant à l'autre pour vendre aux touristes un bracelet de jasmin ou la presse du jour.

Vendeuse de fruits quai Sisowath, face au palais royal, près du débarcadère

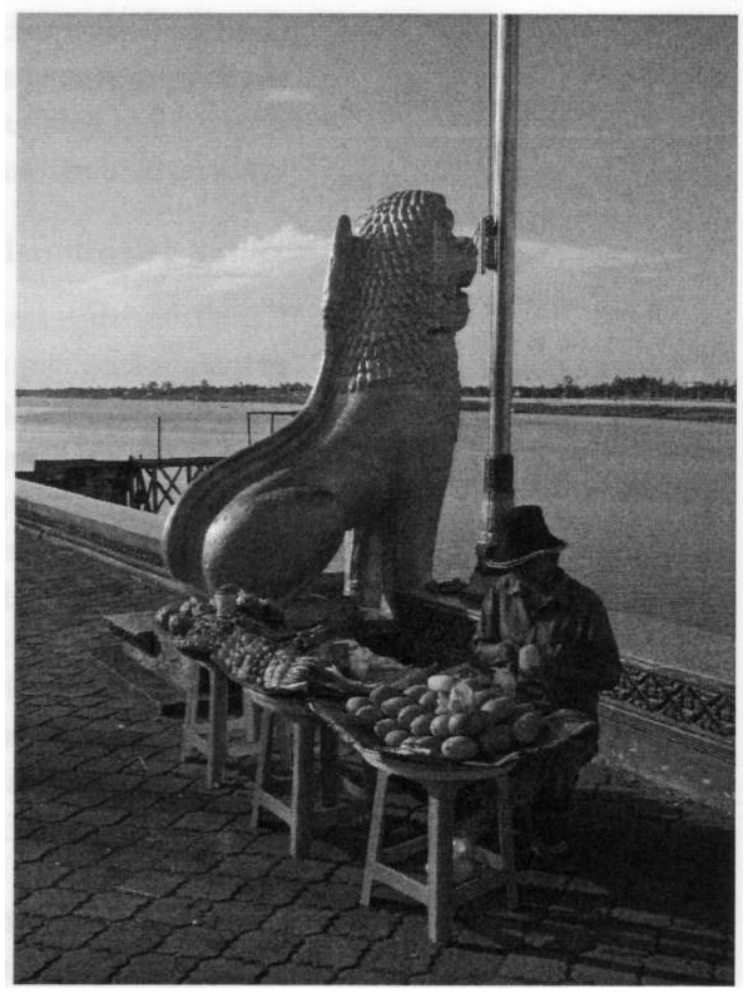

(c) CP, mars 2003.

\section{Entre déterritorialisation et reterritorialisation}

11 Ces territorialités multiples, telles qu'on les perçoit désormais au quotidien ne serait-ce que sur les quais, résultent d'une histoire récente particulièrement heurtée et traumatisante, dans un pays devenu l'un des plus pauvres d'Asie ${ }^{4}$. Du coup d'État du général Lon Nol, provoquant le renversement de Sihanouk le 18 mars 1970, au retrait des troupes vietnamiennes fin 1989, les berges et les espaces récréatifs et de loisirs ont été largement laissés à l'abandon dans la capitale cambodgienne. Entre 1970 et avril 1975, du fait des bombardements américains le long de la frontière avec le Vietnam et de la guérilla qui sévit dans les campagnes, la population de Phnom Penh passe de 600000 habitants à 1,5 million. La capitale doit alors faire face à une multiplication des occupations illégales par un habitat précaire dans les quartiers centraux, due au manque d'équipements en périphérie. 
12 Le 17 avril 1975, les Khmers rouges entrent dans Phnom Penh et vident la ville de sa population en deux jours. Leur programme - désurbanisation, élimination des élites, etc. - ne laisse que quelques milliers de personnes ${ }^{5}$ dans la capitale, jusqu'à sa libération le 7 janvier 1979 par l'armée vietnamienne, et aboutit entre autres à une totale déconstruction des territorialités urbaines, à une "déterritorialisation ", selon l'expression de Marc Bonneville au sujet du développement urbain de l'après-guerre en Europe (Bonneville, 1982) ${ }^{6}$. Les conséquences de cette négation des territorialités urbaines pendant près de cinq ans se font encore sentir à Phnom Penh, réoccupée en grande partie à partir de janvier 1979 par des habitants d'origine rurale. Marcel Roncayolo a défini les «constructions territoriales » comme du «temps consolidé » (1997, p. 19). Un processus lent et complexe de réinstallation de la population dans les villes a lieu au cours des années 1980, alors qu'un embargo international de dix ans contre le gouvernement pro-vietnamien de Phnom Penh, dû à l'occupation militaire du Cambodge par le Vietnam, frappe un pays déjà exsangue. Après la réintroduction du droit de propriété en 1989, de nombreux quartiers informels occupent les interstices laissés aux derniers arrivants et aux populations urbaines les plus pauvres.

13 Dans cette reterritorialisation, l'année 1990 constitue une date charnière : celle de la réorganisation de la fête des eaux (Pierdet, 2005) sur le Tonlé Sap au mois de novembre, alors qu'elle n'avait pas eu lieu depuis vingt ans. La restauration de cette fête traditionnelle qui dure trois jours, ancrée dans un fonds autochtone fait de légendes et de rites agraires, est alors perçue comme une forte volonté de réconciliation nationale. En 1993, le projet urbain, initié par le Gouvernement royal en coopération avec la ville de Paris, qui a consisté à débarrasser les quais Sisowath des entrepôts qui y étaient construits, à redonner aux habitants des espaces de loisirs tournés vers le fleuve, participe de cette dynamique. Une lente reconstruction territoriale prend forme, accompagnée à la fin des années 1990 par le retour des touristes et l'avènement d'une nouvelle société de consommation et de loisirs.

\section{Des pratiques différenciées selon les saisons}

14 La vie qui s'organise autour de cette confluence est fortement marquée par les saisons. Au plus fort de la saison sèche, les quais sont très appréciés des Cambodgiens et des touristes pour leurs espaces ombragés et ventilés. Le soir, quand la température redescend un peu, c'est un endroit agréable et convivial pour se promener en famille. Mais un paroxysme d'affluence a lieu au moment de la fête des eaux qui marque la fin de la saison des pluies et le début de la décrue. Ces festivités que l'on peut, selon Guy Di Méo, assimiler à « un interstice singulier dans l'espace-temps » (2001), sont ici le signal du début d'autres pratiques territoriales. Des milliers de Cambodgiens, venant de tout le royaume, et des touristes se massent le long du fleuve pour observer les joutes nautiques qui participent de la dimension symbolique dont est chargée la confluence. L'arrêt des pluies facilite la tâche aux vendeuses des rues - il leur est plus difficile de travailler pendant la mousson -, tandis que dans les campagnes débute la récolte des riz légers.

De même, les pêcheurs doivent s'accommoder de l'interdiction de pêcher au filet dans les fleuves aux hautes eaux, entre le mois de juin et la fête des eaux. À partir du mois de décembre, au plus fort de la décrue, certaines espèces de poissons migrent en bancs du Grand Lac vers Phnom Penh et le Mékong. Les pêcheurs, le plus souvent des Chams ${ }^{7}$ qui habitent sur les berges ou dans des habitations flottantes, installent alors de grands 
barrages ou dai ${ }^{8} \mathrm{en}$ amont de la capitale. Ils peuvent atteindre $200 \mathrm{~m}$ de long. On en rencontre tout le long du Tonlé Sap jusqu'à Phnom Penh. Ils permettent de capturer des petits poissons de $10 \mathrm{~cm}$ environ qui serviront à la fabrication du prahoc ${ }^{9}$. Les pêches les plus fructueuses ont lieu pendant les pleines lunes des mois de décembre, de janvier et de février. Une nuée de bateaux envahit alors les eaux du Tonlé Sap et la confluence, d'ordinaire quasiment vides.

Pêcheurs à l'embouchure du Tonlé Bassac aux basses eaux.

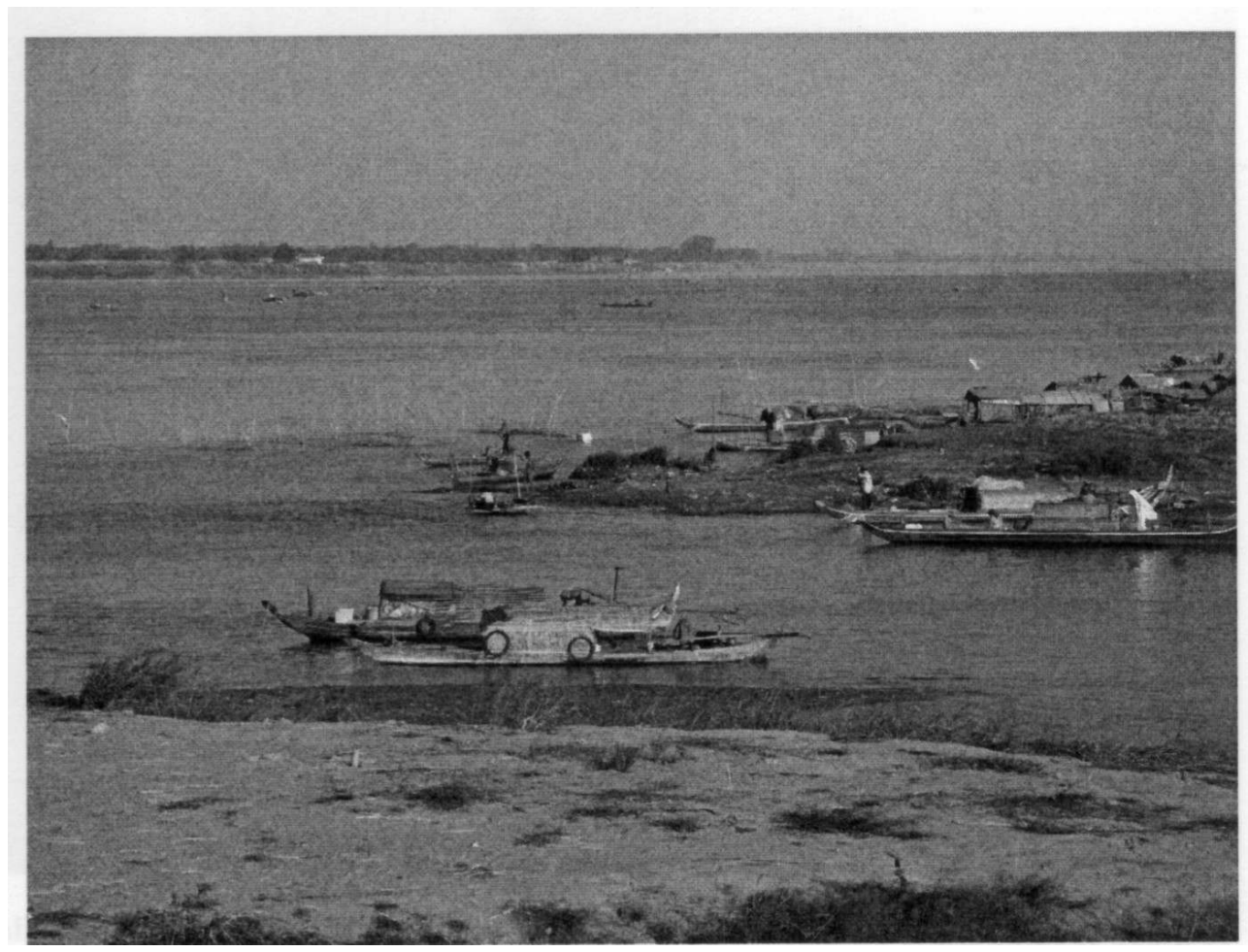

(C) CP,janvier 2003

Les poissons sont alors aussitôt préparés par les Chams pour faire le prahoc qu'ils vendront ensuite aux paysans. Activité saisonnière permettant de survivre quand il n'y a plus rien à manger dans les rizières...

\section{Chruoy Changvar : une presqu'île en cours d'intégration à la ville}

Faisant face à la rive urbanisée de Phnom Penh, entre Tonlé Sap et Mékong, Chruoy Changvar ${ }^{10}$ a longtemps été délaissée par la municipalité. Des rythmes de vie et des pratiques agraires liés à l'eau ont donc perduré jusqu'à il y a peu chez ces populations quasi sédentarisées depuis le début des années 1980. Mais ils apparaissent aujourd'hui fortement menacés.

\section{Répartition des ressources et formes d'occupation différenciées}

18 L'occupation des villages de berge de la presqu'île est assez nettement stratifiée, avec une répartition des activités selon l'origine ethnique. On la rencontre tout le long des 
voies fluviales au Cambodge. Comme Jean Gallais l'a constaté au sein du delta du Niger (1967, p. 105-117) dans les années 1960, plusieurs groupes ethniques cohabitent au sein du même village, entretenant une parfaite complémentarité des activités pratiquées par chacun d'eux. D'après le chef de village, environ 200 familles chames de nationalité cambodgienne et 70 familles vietnamiennes vivent à l'extrémité méridionale et sur la partie orientale de la presqu'île. Dans cette partie de la capitale, rythmée par le mouvement des eaux, on ne parle pas du Mékong ${ }^{11}$, mais du Tonlé Thom ou Grand fleuve. Cette rive est fortement soumise à érosion. La partie supérieure de la berge est donc abrupte, occupée par un habitat sur pilotis dans lequel vivent des Chams. La partie médiane voit se reconstruire chaque année des paillotes, emportées à la montée des eaux, tandis qu'au ras de l'eau sont accostées des barques de pêche des Vietnamiens et des Chams.

Familles chames sur la berge orientale de Chruoy Changvar.

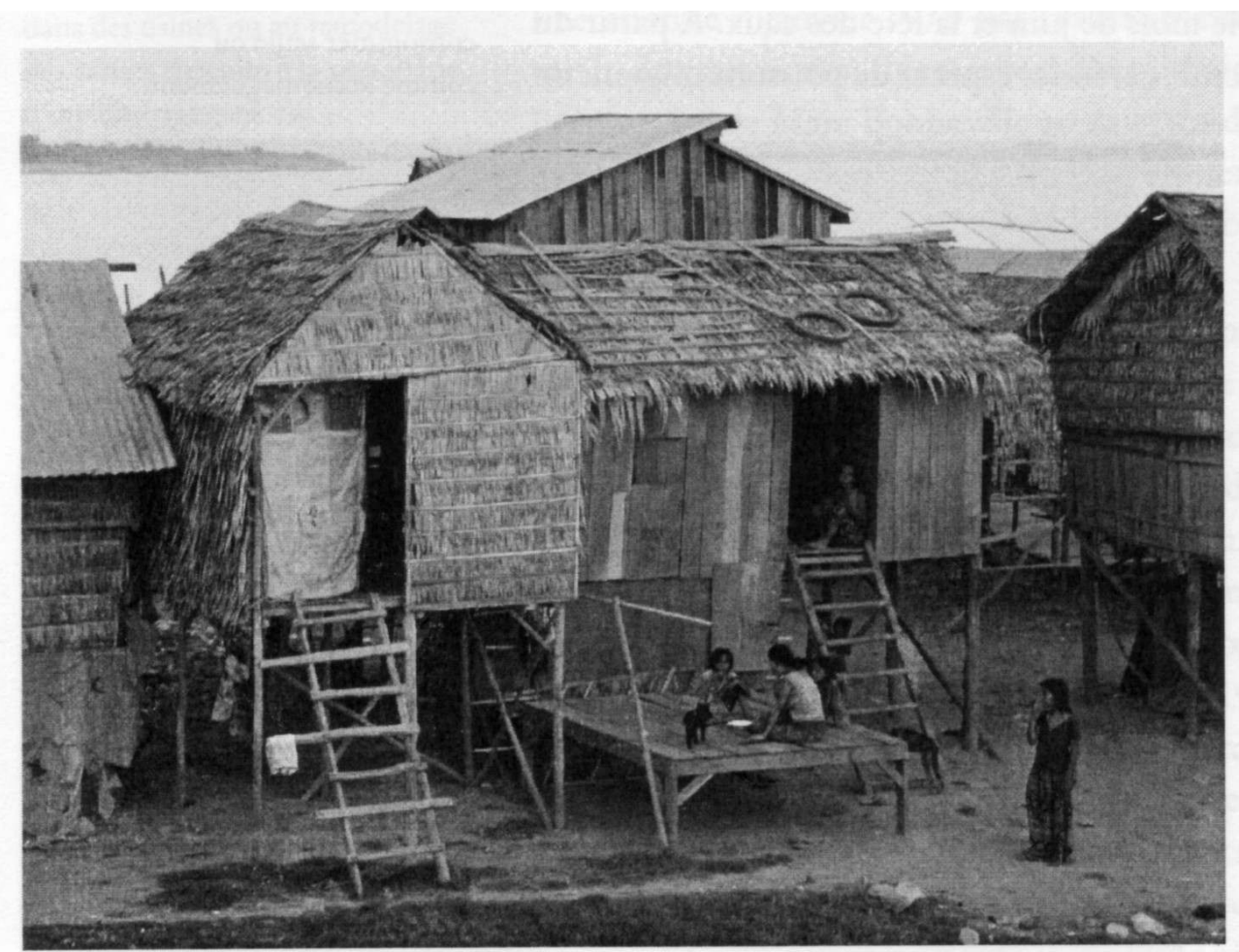

(c) CP, mars 2004

19 Une grand-mère chame, assise sur les marches de l'escalier de sa maison en bois, explique qu'elle habite ici depuis la chute du régime de Pol Pot. Elle y vivait déjà au début des années 1970. Comme presque tous ici, elle ne dispose pas de titre de propriété foncière. Dans une famille vietnamienne, une vieille femme vivait elle aussi ici avant Pol Pot. En avril 1975, elle a pu s'enfuir en bateau au Vietnam et est revenue au Cambodge en 1979. Mais son ancienne maison avait déjà été réoccupée. Dans sa famille, les plus vieux vont vendre des fruits et des gâteaux au marché, dans le centre de la ville, tandis que les garçons plus jeunes sont maçons et travaillent sur les chantiers de construction. Ils vendent de même le produit de la pêche au marché. Mais comme beaucoup de familles vietnamiennes, elle pratique surtout l'élevage du poisson sous la maison, dans une cage de plusieurs mètres cubes à laquelle on accède par une trappe 
située dans le plancher, qui permet de nourrir et de récupérer les poissons ${ }^{12}$. Mais à la différence des Chams et des Khmers, les Vietnamiens de Chruoy Changvar ne participent pas à la fête des eaux.

Ces activités, qui reposent sur une répartition des ressources du fleuve et des berges entre les ethnies, engendrent des formes d'occupation différenciées. Les bateaux accostés plus bas permettent de pratiquer une pêche familiale au filet. Ne cultivant pas la terre, les Chams font du troc avec les Khmers qui cultivent un petit potager un peu plus loin. Ils vendent le poisson directement au phsar Cha - ou vieux marché - ou à un acheteur qui le revendra sur un autre marché en dehors de la ville. Le bateau leur sert aussi de refuge aux hautes eaux, saison où seule la pêche à la ligne pour une consommation familiale est autorisée.

21 Une vingtaine de familles vivent sur la rive bordée par le Tonlé Sap. De jeunes Chams s'affairent à la réparation du fond d'une barque de pêche, objet de toutes les attentions. Selon un proverbe, "si la matière de la barque est entièrement belle, elle est propice à la fortune [...] » (Bitard, 1955, p. 159). Ils occupent cette partie de la berge depuis qu'ils sont sortis d'un camp de travail situé près de Battambang en 1979. Cet homme vit dans un bateau accosté à la berge avec sa famille. Il n'occupe donc aucune parcelle de terre et se trouve ainsi marginalisé. Il n'en est pourtant pas moins territorialisé : il vit de la pêche et du commerce qu'il effectue au marché. Les filets sont lancés en travers du fleuve le soir vers 5-6h00 et relevés vers 3-4h00 du matin. Le poisson est aussitôt mis dans des paniers, sorti des bateaux et porté au marché.

Alignement de barques de pêcheurs chams, au bord du Tonlé Sap.

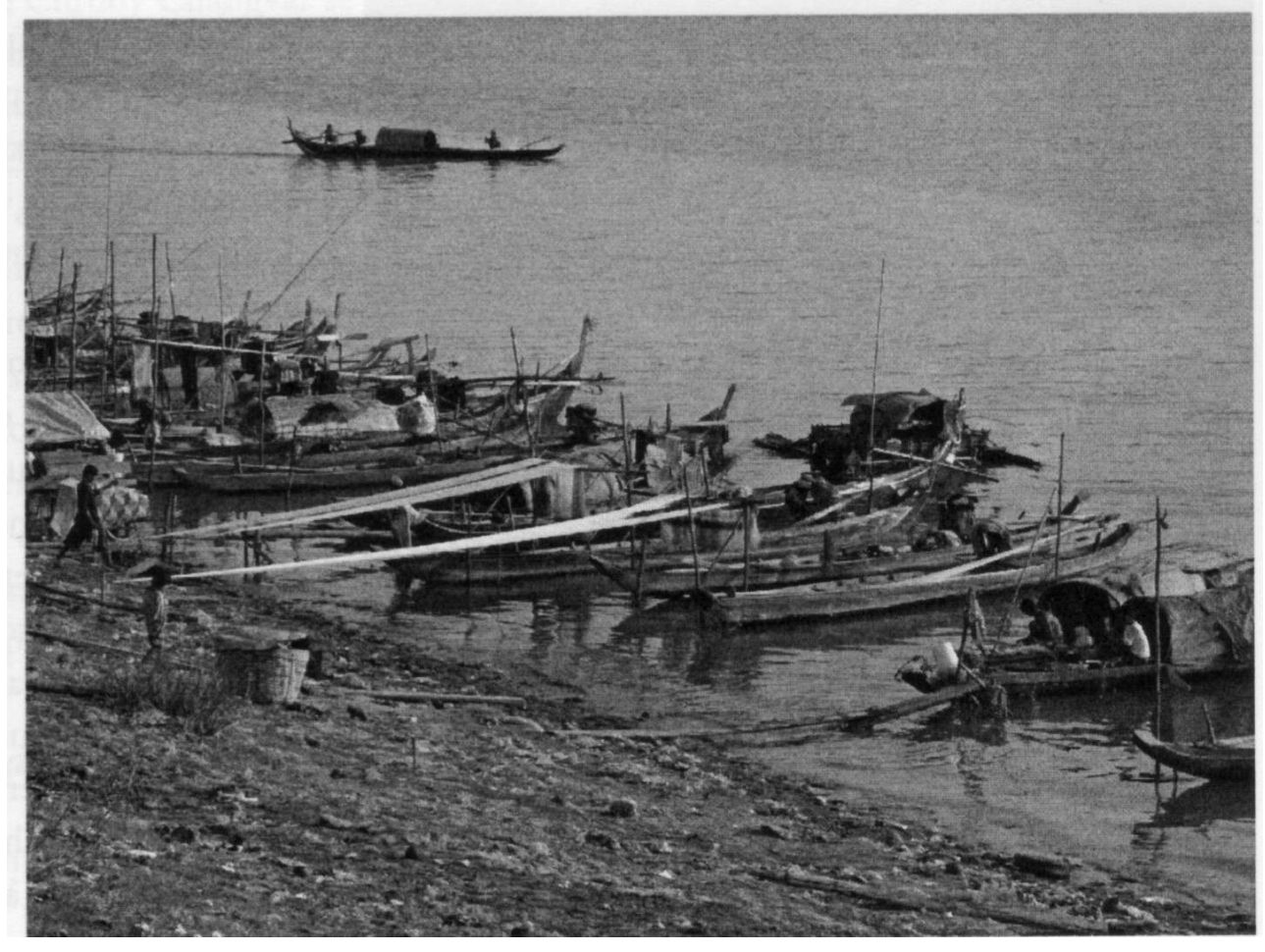

(c) $\mathrm{CP}$, mars 2004 


\section{Territorialités fluctuantes et dimension symbolique}

22 À la différence des Chams, les Khmers cultivent les berges. Une dizaine de rayons de légumes ont ainsi été mis en culture depuis le retrait des eaux, sur les limons déposés dans la partie basse de la berge faisant face à Phnom Penh, qui est marquée par un net processus d'accumulation. Le temps est compté jusqu'en juin juillet, donc aucune place n'est perdue. Ces territorialités sont temporaires et fluctuent au rythme des saisons : aux hautes eaux, ces terres fertiles sont recouvertes par 6 ou $7 \mathrm{~m}$ d'eau. D'après une jeune mère de famille, trois demi-rangées sont attribuées à chacune des familles khmères vivant dans la presqu'île, près du Vatt Prah Chum Sako. Cette pagode, fondée à la fin du XIXe siècle, est située plus en retrait de la berge que le Vatt Botiyaram, qui est visible du quai Sisowath. Sous Pol Pot, elle a été fermée mais n'a pas été détruite. Contrairement aux Vietnamiens, les familles khmères de la presqu'île accordent une grande importance à la fête des eaux, ainsi qu'aux trois pirogues de course conservées sous un abri situé dans l'enceinte du Vatt Prah Chum Sako.

Dans la plupart des villages situés près d'un cours d'eau, la pagode possède au moins une pirogue de course qui, par extension, représente le village. Ces pirogues portent chacune un nom, souvent féminin. Ici cette jeune femme peut les citer de mémoire: «la belle mer » ou pisei serei sako, « la fleur de la capitale» ou bopha rich thea ni, et « le pouvoir de l'enfant » ou koma mean rith. Elle assure qu'un esprit ${ }^{13}$ ou bray habite bien ces pirogues, et que lui seul peut les faire gagner. Deux d'entre elles ont déjà remporté une course, ce qui leur vaut une grande notoriété qui retentit sur toute la communauté villageoise. Ces pirogues sont surélevées au-dessus du sol, sous un hangar « ouvert aux quatre vents ». Son esprit est tellement redouté que « les femmes enceintes ne peuvent même pas passer devant de peur de faire une fausse couche » (Ang Chouléan, 1986, p. 138).

24 Assimilées à des êtres vivants, les pirogues sont toujours très décorées. Dans ce processus de personnification, on les dote de cheveux et de deux yeux à l'avant. Ici, ils ont été laissés sur les pirogues alors que, normalement, il convient de les enlever. De même, leur nom est gravé sur le côté, avec celui du village. Ces villageois sont particulièrement fiers que la pagode possède ces pirogues, alors qu'il arrive désormais que leur utilisation soit dévoyée de son sens au profit d'une personnalité politique ou d'une entreprise. 
Pirogue de course décorée, abritée sous un hangar ouvert, au Vatt Prah Chum Sako.

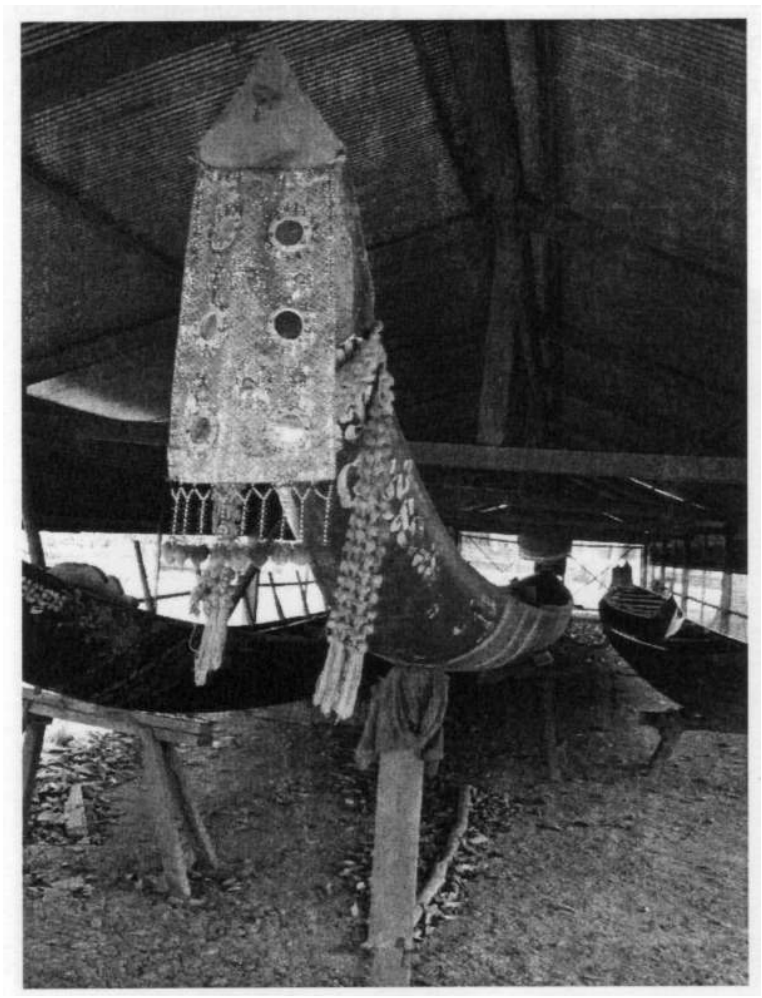

(c) CP, mars 2004

\section{Projet urbain et citadinité}

La presqu'île est caractérisée par une progradation vers le sud due à l'accumulation de sédiments à son extrémité. Dans le but de l'enrayer, et pour des raisons d'ordre symbolique (Pierdet, 2005), la municipalité a entrepris en 2002 de faire déplacer aux basses eaux les limons accumulés sur la partie occidentale et méridionale de la presqu'île, puis de construire un parapet et des quais. Pendant les travaux, ces populations au mode de vie agraire, toutes ethnies confondues, se sont déplacées sur une partie des berges qui n'était pas concernée par le projet. Une fois le projet achevé, elles se sont réinstallées là où elles étaient auparavant, depuis le début des années 1980.

[Cet exemple vient illustrer les propos de Maryvonne Le Berre pour qui] toute action d'un groupe passe par la médiation de la matérialité territoriale : le groupe social, acteur du système spatial, produit le territoire, s'y maintient et s'y reproduit; le territoire, lieu de vie du groupe, lui fournit les conditions de ce maintien et de cette reproduction : c'est donc aussi un acteur du système spatial (1992, p. 634).

Autrement dit, les populations de Chruoy Changvar se sont maintenues d'une part parce qu'elles sont en interrelations étroites entre elles et avec le territoire qui leur fournit les conditions de leur maintien; d'autre part, parce qu'elles contribuent à la reproduction du territoire dont elles ont besoin.

L'aménagement de ces quais, de l'éclairage public, des espaces verts, puis d'une route depuis le pont qui franchit le Tonlé Sap a incité les habitants de la rive urbanisée de Phnom Penh à venir dans la presqu'île pour dîner ou pour profiter de la vue sur le palais royal illuminé. Les jeunes s'y promènent en groupes. Le transfert de ces 
pratiques citadines dans cet espace encore totalement rural il y a peu, a donc suscité des stratégies d'adaptation - déplacement momentané, moindre pratique de la pêche dans l'axe du palais royal - de la part de ces populations agraires dans l'espoir d'y rester encore longtemps. Mais il s'agit sans doute de modes de vie en sursis, tout du moins sur la berge occidentale bordée par le Tonlé Sap.

\section{Une recomposition des territorialités liées à l'eau à Phnom Penh}

Depuis le début des années 2000, ces multiples territorialités liées à l'eau connaissent une recomposition accélérée, qui fait émerger des conflits d'usages importants à l'échelle de la capitale.

\section{Les quais entre habitat, commerce et tourisme}

Sur les quais se pose désormais la question de savoir comment faire coexister, sur cet espace linéaire, la zone touristique la plus attractive de la capitale, les activités qu'elle engendre, et les pratiques traditionnelles des Phnompenhois. Les lieux d'accueil pour les touristes - assurant hébergement et restauration - sont surtout situés dans cette zone centrale de la capitale. Ils n'y restent souvent que deux ou trois jours, le temps de découvrir les principaux édifices situés à proximité, ainsi que Tuol sleng, l'ancien lycée devenu prison. Phnom Penh est largement délaissée par les touristes au profit de Siemreap. Mais la concentration touristique sur les quais est aujourd'hui très forte. Pendant la période de l'Apronuc ${ }^{14}$, seul un établissement sur pilotis surplombant le Tonlé Sap était ouvert : le Kirirom, aujourd'hui nommé le Gold Fish River, était l'un des seuls lieux animés où sortir le soir, avec son bar circulaire au centre de la pièce, et une large vitre donnant sur le fleuve. D'autres établissements l'ont rejoint depuis, surtout fréquentés par les Cambodgiens. Le bâti connaît aussi une forte évolution: des villas coloniales sont démolies; le compartiment chinois traditionnel des quais, construit dans une parcelle tout en longueur, et étroit en façade, associant commerce au rez-dechaussée et logement à l'étage, souvent tenu par des familles d'origine chinoise, est aujourd'hui supplanté par des grands hôtels. 
Alignement de compartiments chinois sur le quai Sisowath

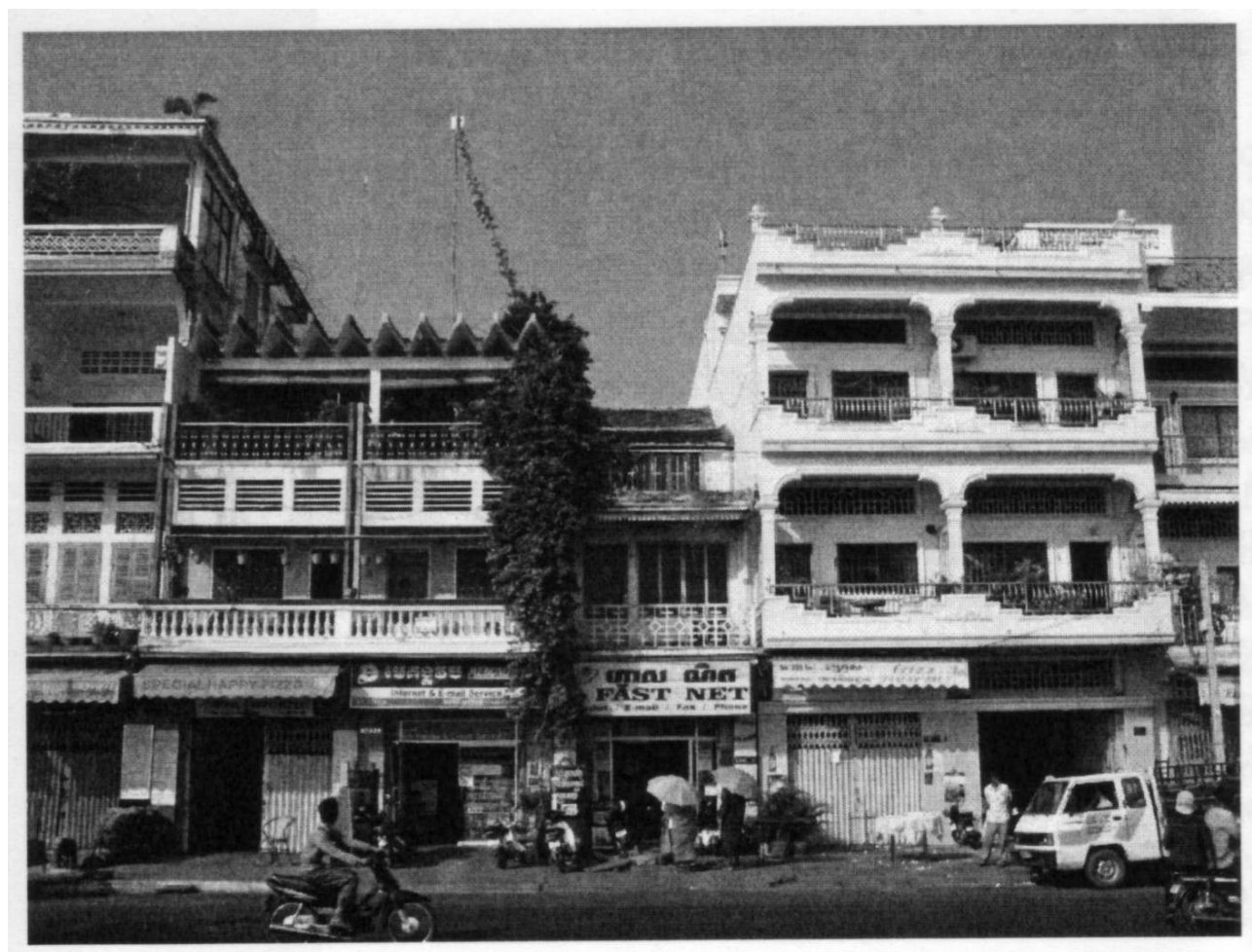

(C) CP, mars 2004.

30 Après rachat de compartiments mitoyens, les bâtiments sont démolis et reconstruits selon une autre configuration, comme dans le cas de l'hôtel-restaurant K-West, tenu par un Français.

31 Un peu plus au sud, une entreprise de pompes funèbres chinoises voisine encore avec des pizzerias proposant diverses formules de happy pizzas. Cafés internet et hôtelsrestaurants se succèdent. Les chambres avec vue sur le Tonlé Sap et la confluence sont très prisées par les touristes. Ces lieux d'hébergement occupent désormais quasiment tout l'espace jusqu'aux embarcadères des bateaux en partance pour Siemreap ou le Vietnam, et se livrent une concurrence féroce. La location d'un étage dans la zone centrale des quais est estimée à $700 \$$ par mois. À l'achat, les prix monteraient à $200000 \$$ avec le bâtiment, ce qui constitue un investissement difficile à rentabiliser. 
Hôtel-restaurant le K-West, quai Sisowath, construit en 2003.

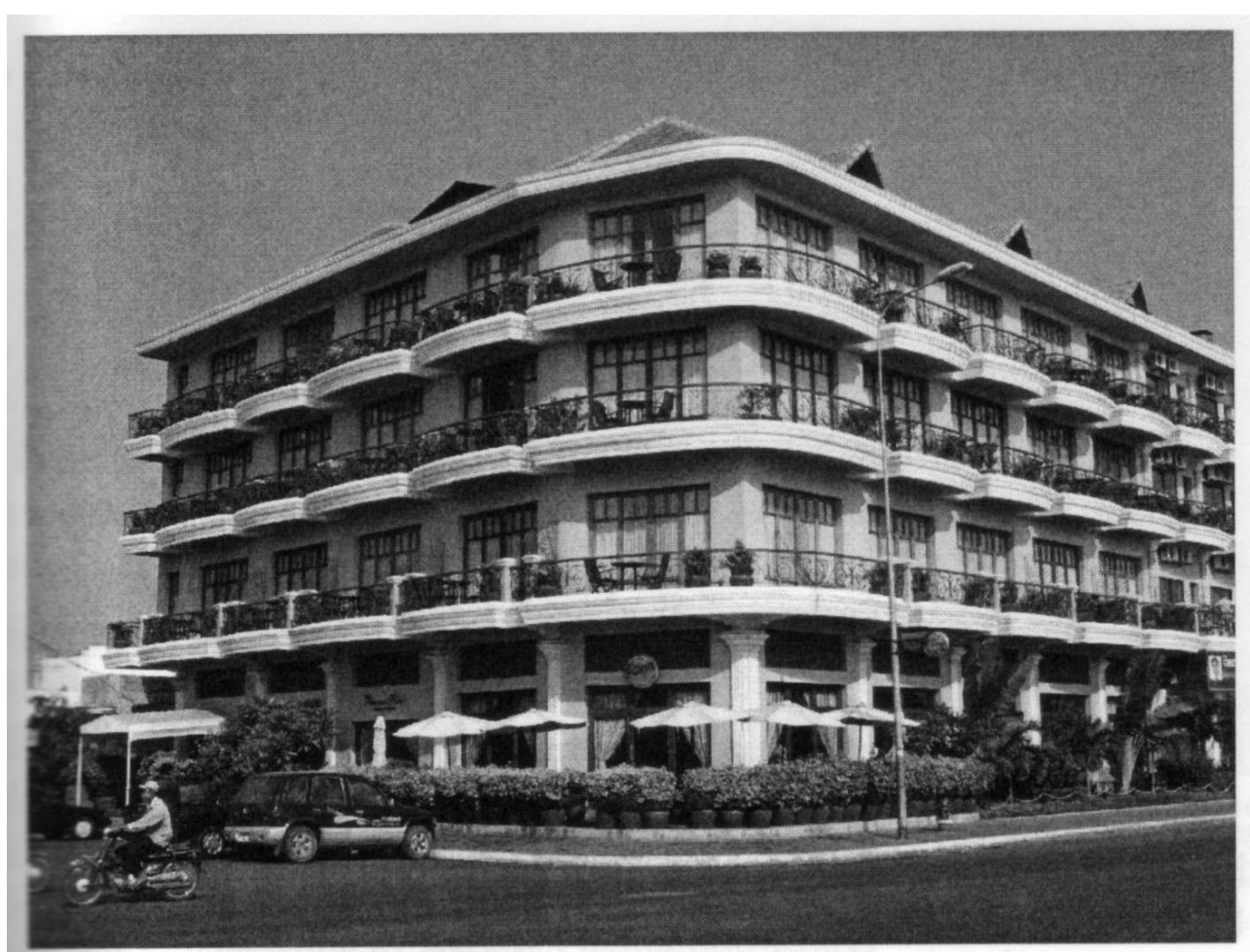

(c) CP, mars 2004

\section{Koh Pich ${ }^{15}$ : la disparition d'un jardin maraîcher face à l'affirmation des modèles internationaux}

Au début de la saison sèche, lorsque le niveau des eaux du Mékong s'abaisse et que le Bassac rentre dans son lit, une langue de terre émerge à la jonction des deux cours d'eau : 70 ha enrichis de limon par cette longue immersion réapparaissent. Jusqu'en 2005, 134 familles, dotées de titres de propriété pour la plupart, cultivaient une cinquantaine de variétés de légumes sur ces lopins. Le chef de ce village occupait ces terres six mois par an depuis les années 1980. Elles approvisionnaient surtout les marchés de la capitale. Avant l'aube, les femmes partaient vendre la production de la veille: liserons d'eau, haricots verts, choux, etc. Les familles gagnaient de 20000 à 30000 riels par jour, soit 5 à 6 dollars. Une partie des récoltes était aussi convoyée dans les villes portuaires du pays, sur le littoral. Un village flottant de pêcheurs chams jouxtait l'île à l'embouchure du Bassac. 


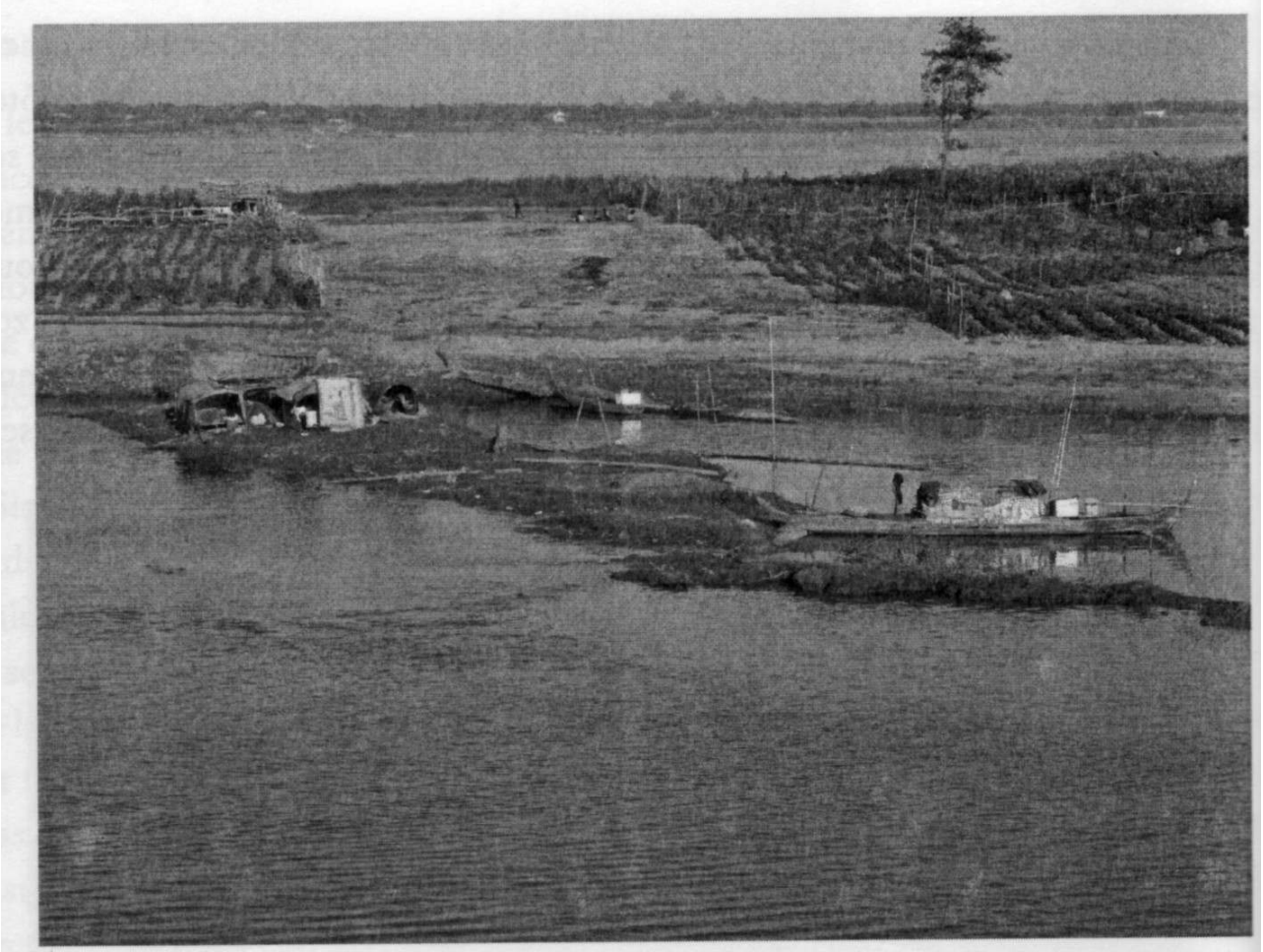

(c) CP, janvier 2003.

33 Les habitants de Koh Pich ont depuis été expulsés au profit d'un projet de gated community, supporté par la Canadia Bank, associé à la réalisation de nouveaux ponts sur le Tonlé Bassac. L'île est endiguée et remblayée. Ceci vient illustrer l'actuelle recomposition de la centralité urbaine autour de la confluence par l'intégration dans des projets urbains d'espaces jusqu'alors laissés à des activités à faible valeur ajoutée. Les parcelles ont été rachetées par la municipalité à leurs propriétaires à bas prix ou échangées contre des parcelles en périphérie de la ville, éloignées du territoire dont ces populations vivaient et que, réciproquement, elles entretenaient.

\section{Le remblai des lacs à la périphérie de la ville}

En périphérie de Phnom Penh, de vastes superficies inondables, des beng ou lacs, permettent d'absorber de grandes quantités d'eau déversées par des prek ou chenaux, à partir des voies fluviales, en arrière du bourrelet de berge au plus fort de la crue. À la saison sèche les eaux se retirent alors par gravité et par évaporation, laissant des terres fertiles prêtes à cultiver et sur lesquelles faire paitre les troupeaux. Les mares qui subsistent servent d'abreuvoir aux betes, mais aussi de réserve pour les paysans: poissons pris au piège, escargots, fleurs de lotus, etc.

Mais ces espaces sont aujourd'hui l'objet d'une forte spéculation de la part de promoteurs. Dès qu'un projet de route est annoncé, des projets immobiliers se multiplient dans la zone concernée. Or ces terrains inondables ne peuvent être urbanisés qu'après avoir été remblayés. Pour ce faire, d'importantes carrières sont creusées à proximité afin de permettre ces remblais désordonnés sur plusieurs centaines d'hectares, modifiant à tout jamais les lieux de vie traditionnels des paysans 
qui vivaient dans la périphérie. Rares sont ceux qui disposent de titres fonciers à négocier avec les promoteurs ou avec les okñà, des personnages puissants qui achètent leur charge. D'autre part, tant que le service du Cadastre n'a pas clairement délimité ces espaces lacustres, ils n'ont pas d'existence juridique formelle. Le beng Trabek, situé dans les districts centraux de la capitale, au sein du tissu urbain, a ainsi pu être conservé par la municipalité après avoir été officiellement délimité et cartographié. Son rôle de réceptacle des eaux usées et pluviales a été maintenu.

Une fois remblayés, ces lacs sont vendus avec une forte plus-value, comme cela a déjà été le cas pour beaucoup d'entre eux. Le quotidien Cambodge Soir relate ainsi comment le beng Kbal Damrei est en passe de disparaître ${ }^{16}$. Sous la houlette d'un haut gradé des Forces armées royales khmères (FARK), le général Ke Kim Yan, les environs du lac ont été interdits d'accès au printemps 2005. Puis des activités de remblaiement ont débuté, avec la mise à contribution de militaires. Mais la municipalité de Phnom Penh a alors obligé l'officier à réaliser des infrastructures de drainage des eaux de ruissellement afin d'éviter que les populations alentours ne subissent les inondations qu'aurait nécessairement engendrées ce projet puisque, une fois remblayé, aucun lac ne permet plus d'absorber l'excès d'eau. Les travaux ont ensuite pu reprendre. Des grilles entourent désormais le site sur lequel un golf pourrait être réalisé.

Remblaiement du beng Poung Peai, au nord de Phnom Penh.

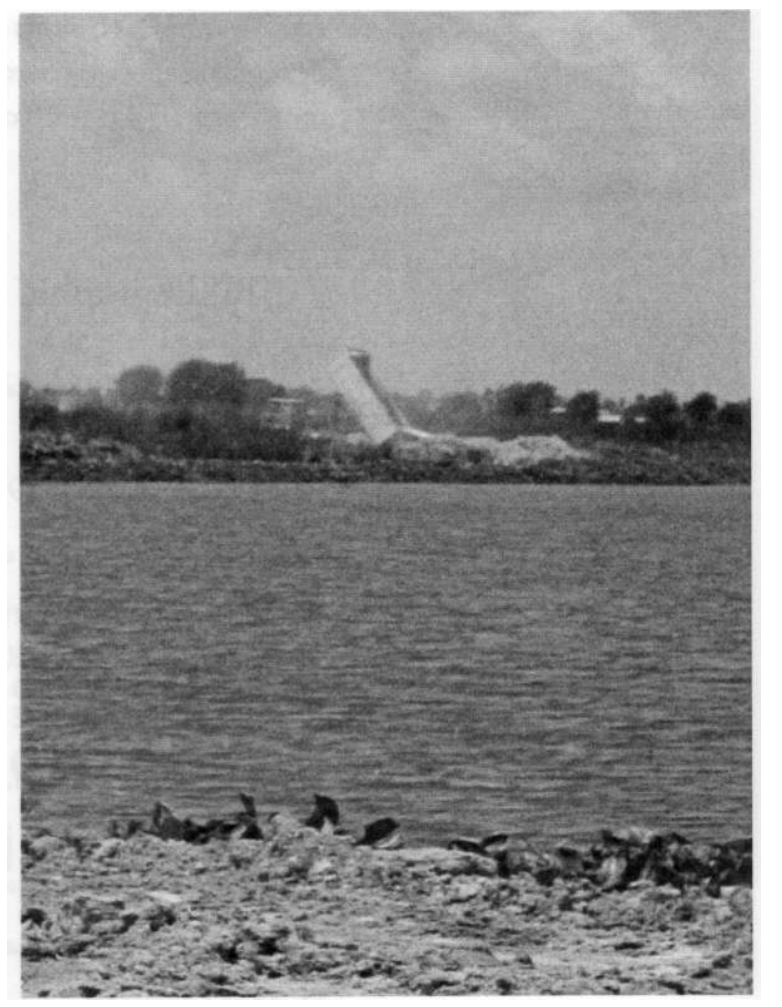

(C) CP, septembre 2003.

37 Au total, les formes de territorialités en rapport avec l'eau, lentement reconstruites à Phnom Penh depuis le début des années 1980, sont aujourd'hui mises à mal par un processus sous-tendu par une volonté de modernité. Les modes de vie agraire sont de plus en plus niés et les berges de la partie méridionale de la confluence, désormais gagnées par une urbanisation accélérée, tendent à devenir des vitrines pour les 
investisseurs internationaux. Cette capitale fluviale d'Asie des Moussons à l'équilibre hydraulique fragile et encore imparfaitement retrouvé, qui avait jusqu'alors laissé coexister des territorialités très différenciées, en particulier sur les berges, semble aujourd'hui s'orienter vers une banalisation de ces espaces les plus touristiques de la capitale. Mais les conflits d'usages qui se multiplient, avec en arrière fond les problèmes de régularisation foncière des plus pauvres, donnent au contraire l'image de la mise en cuvre brutale d'une déconstruction de territorialités lentement sédimentées.

\section{BIBLIOGRAPHIE}

Ang Chouléan, 1986, Les êtres surnaturels dans la religion populaire khmère, Paris, Cedorek, 349 p.

Ang Chouléan, 1987-1990, « Le sacré au féminin », in Seksa Khmer, 10-13, 30 p.

Apur, 1997, Phnom Penh, développement urbain et patrimoine, Paris, $160 \mathrm{p}$.

Bailly A., 1982, "Territorialité urbaine : réalité ou mythe des sociétés productivistes? », in

Géopoint 82 : Les territoires de la vie quotidienne, Groupe Dupont.

Bitard P., 1955, « Traité de l'achat des barques », BSEI, XXX, n² 2, p. 157-162.

Cambodge Soir, quotidien, $1^{\mathrm{er}}$ au 2 avril 2005.

Bonneville M., 1982, « Territoires et territorialité en milieu urbain », in Géopoint 82 : Les territoires de la vie quotidienne, Groupe Dupont, p. 357-369.

Delvert J., 1961, Le paysan cambodgien, Paris, Imprimerie nationale, 742 p.

Di Meo G., 2001, « Le sens géographique des fêtes », Annales de géographie, n 622, p. 624-646.

Gallais J., 1967, Le delta intérieur du Niger. Étude de géographie régionale, Dakar, IFAN, 2 tomes.

Le Berre M., 1992, « Territoires », in Bailly A., Ferras R., Pumain D. (dir.), Encyclopédie de géographie, Paris, Economica, p. 617-638.

Morelle M., Fournet-Guérin C., 2006, « Les nuits tananariviennes : citadinités et marginalités en construction ", Cybergeo, $\mathrm{n}^{\circ} 342,28$ juin.

Pierdet C., 2005, «La symbolique de l'eau dans la culture cambodgienne - Fête des eaux et projets urbains à Phnom Penh ", Géographie et cultures, n 56, hiver, p. 5-22.

Roncayolo M., 1997 (rééd.), La ville et ses territoires, Paris, Gallimard, 280 p.

\section{NOTES}

1. Le terme khmer tonlé signifie "fleuve ». Cette retranscription phonétique est destinée à faciliter la lecture.

2. D'après le dernier recensement intermédiaire de 2004, la population urbaine du Cambodge était estimée à 2,5 millions d'habitants pour une population totale de 13,5 millions. Phnom Penh 
regroupait alors 1,3 million d'habitants, soit environ $50 \%$ de la population urbaine totale (Institut national de la statistique).

3. Au cours d'un séjour de quinze mois effectué entre août 2002 et mars 2004, les observations ont été complétées par des entretiens ouverts réalisés sur les quais avec différents acteurs propriétaires de restaurants, touristes, joggers matinaux, loueurs d'embarcations -, ainsi que par une quarantaine d'enquêtes menées dans deux villages de la presqu'île et dans l'île située à l'embouchure du Tonlé Bassac.

4. En 2000, le Cambodge se situe, d'après le Programme des Nations unies pour le développement (PNUD), au $130^{\mathrm{e}}$ rang mondial sur 173 pays classés, avec un Indice de développement humain (IDH) de 0,543.

5. Il s'agit de dirigeants Khmers rouges, des prisonniers des différents camps situés dans la ville, de Cambodgiens employés dans des usines ou au remodelage des casiers rizicoles à la périphérie, d'invités étrangers, etc.

6. Dans ce contexte, M. Bonneville désigne ainsi la "dislocation des «équilibres" sociorésidentiels", le transfert massif de populations des campagnes vers les villes, le remodelage des tissus urbains, etc.

7. Ou «Khmer Islam» sont de nationalité cambodgienne mais de religion musulmane. On les rencontre surtout le long des berges du Mékong et du Tonlé Sap où ils vivent de la pêche et du commerce.

8. Le daï est un piège fixe tenu par des radeaux de bambou ancrés, formant des sections de $25 \mathrm{~m}$ de long sur $10 \mathrm{~m}$ de profondeur. Les mailles sont de $10 \mathrm{~cm}$ à l'entrée du piège et de $10 \mathrm{~mm}$ à l'extrémité, où un panier de rotin est fixé.

9. Les poissons sont étêtés et vidés sur les berges, puis entassés dans des paniers en bambou tressé et piétinés pour être réduits, avant d'être mélangés à du sel. Après séchage au soleil, cette pâte de poisson fermenté, qui dégage une très forte odeur, est achetée par les Khmers qui la conservent dans des jarres avant de la consommer seule ou comme accompagnement.

10. Le terme chruoy signifie « avancée de terre dans l'eau » ou presqu'île.

11. L'étymologie khmère du terme Mékong est encore soumise à discussion. Ce terme a été imposé par les Occidentaux pour désigner cette voie fluviale. On l'apprend à l'école, on l'utilise dans des documents administratifs, mais on ne l'emploie pas dans le langage courant, en dehors du centre de Phnom Penh.

12. De nombreux villages flottants de ce type existent dans le delta du Mékong. Des visites touristiques y sont fréquemment organisées à partir de Ho-Chi-Minh-Ville.

13. Nos remerciements à l'ethnologue Ang Chouléan pour les entretiens qu'il nous a accordés en février-mars 2004 et ses explications passionnantes au sujet des pirogues de course et des esprits qui les habitent. Voir Ang Chouléan, 1987-1990.

14. L'Autorité provisoire des Nations unies au Cambodge (Apronuc) a été créée le 28 février 1992 et son mandat a pris fin le 24 septembre 1993. Son rôle était de garantir l'application de l'accord de paix signé à Paris le 23 octobre 1991.

15. Le terme koh signifie « île »; Koh Pich est littéralement « l'île au diamant ».

16. Quotidien Cambodge Soir, $1^{\mathrm{er}}$ au 2 avril 2005. 


\title{
RÉSUMÉS
}

Du fait de la singularité de son site, la capitale fluviale du Cambodge a vu se développer des pratiques territoriales liées à l'eau d'une grande diversité. Des territorialités qui trouvent leurs racines dans un mode de vie agraire, caractéristique de sociétés riveraines des grands fleuves de l'Asie des Moussons, coexistent avec des pratiques citadines plus récentes à Phnom-Penh. Alors qu'elles ont participé à la lente reconstruction territoriale mise en œuvre depuis le début des années 1980, à la suite du génocide, ces pratiques agraires sont aujourd'hui mises à mal au profit de pratiques citadines plus conformes à l'image de modernité qu'entend donner à voir la capitale d'un pays en plein renouveau, auprès des investisseurs et des touristes.

As a result of its singular setting as a fluvial town, the capital city of Cambodia witnessed the development of extremely varied territorial practices in connection with water. Territorialities with deeply-rooted agrarian ways of life, which is typical of societies living along the large rivers of Monsoon Asia, coexist with more recent urban practices in Phom Penh. Even though agrarian practices contributed to the slow territorial reconstruction initiated in the early 80 's, after the genocide, they are now losing out to urban practices more consistent with the image of modernity that the capital city of a newly-born country wants to project to investors and tourists.

\section{INDEX}

Mots-clés : quotidien, territoire, agriculture, espace urbain, espace rural, tourisme, eau Index géographique : Cambodge

\author{
AUTEUR \\ CÉLINE PIERDET \\ Doctorante en géographie, \\ Ladyss, Université de Paris 1, Panthéon-Sorbonne, \\ 191 rue Saint-Jacques, 75005 - Paris \\ c_gedeon2000@yahoo.fr
}

